\title{
Colostrum avoidance, prelacteal feeding and late breast-feeding initiation in rural Northern Ethiopia
}

\author{
Nikki L Rogers ${ }^{1}{ }^{*}$, Jemilla Abdi ${ }^{2}$, Dennis Moore ${ }^{1}$, Sarah Nd'iangui ${ }^{1}$, Linda J Smith ${ }^{3}$, \\ Andrew J Carlson ${ }^{4,5}$ and Dennis Carlson ${ }^{4}$ \\ 'Department of Community Health, Substance Abuse Resources and Disability Issues (SARDI) Program, \\ Wright State University Boonshoft School of Medicine, 3171 Research Boulevard, Room 259, Dayton, \\ $\mathrm{OH}$ 45420-4006, USA: ${ }^{2}$ Department of Midwifery, College of Medicine and Health Science, University of \\ Gondar, Gondar, Ethiopia: ${ }^{3}$ Bright Future Lactation Resource Centre Ltd, Dayton, OH, USA: ${ }^{4}$ Kossoye \\ Development Project, Bainbridge Island, WA, USA: ${ }^{5}$ Department of Political Science, Capital University, \\ Columbus, $\mathrm{OH}$, USA
}

Submitted 24 March 2010: Accepted 9 January 2011: First published online 21 April 2011

\begin{abstract}
Objective: To identify specific cultural and behavioural factors that might be influenced to increase colostrum feeding in a rural village in Northern Ethiopia to improve infant health.

Design: Background interviews were conducted with six community health workers and two traditional birth attendants. A semi-structured tape-recorded interview was conducted with twenty mothers, most with children under the age of 5 years. Variables were: parental age and education; mother's ethnicity; number of live births and children's age; breast-feeding from birth through to weaning; availability and use of formula; and descriptions of colostrum $v$. other stages of breast milk. Participant interviews were conducted in Amharic and translated into English.

Setting: Kossoye, a rural Amhara village with high prevalence rates of stunting: inappropriate neonatal feeding is thought to be a factor.

Subjects: Women (20-60 years of age) reporting at least one live birth (range: $1-8$, mean: $\sim 4)$.

Results: Colostrum (inger) and breast milk (yetut wotet) were seen as different substances. Colostrum was said to cause abdominal problems, but discarding a portion was sufficient to mitigate this effect. Almost all (nineteen of twenty) women breast-fed and twelve (63\%) reported ritual prelacteal feeding. A majority (fifteen of nineteen, 79\%) reported discarding colostrum and breast-feeding within $24 \mathrm{~h}$ of birth. Prelacteal feeding emerged as an additional factor to be targeted through educational intervention.

Conclusions: To maximize neonatal health and growth, we recommend culturally tailored education delivered by community health advocates and traditional health practitioners that promotes immediate colostrum feeding and discourages prelacteal feeding.
\end{abstract}

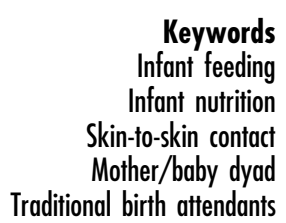

The feeding of newborn infants has important implications for immediate and future health, especially in developing countries such as Ethiopia that have high rates of malnutrition, infectious diseases and mortality for children under the age of 5 years $^{(1,2)}$. Exclusive breast-feeding from birth through 6 months of age has long-term health and emotional benefits for both mother and child and is associated with lower infant morbidity and mortality and better growth ${ }^{(3)}$. Given the health risks associated with artificial (formula) feeding and ineffective breast-feeding, WHO and UNICEF developed a strategy to underscore the importance of exclusive and maximal breast-feeding, summarized in their guide for practitioners entitled Ten Steps to Successful Breastfeeding ${ }^{(4,5)}$. These steps include educating mothers about breast-feeding management; helping mothers and babies initiate breast-feeding within 30 min of giving birth; and prohibiting prelacteal feeding (feeding any other substance before first breast-feeding) or complementary feeding any substance other than breast milk before the age of 6 months.

Colostrum is a thick yellow/orange breast milk that is the earliest and most immunologically protective secretion of the mammary gland during lactogenesis $I^{(6,7)}$. It is highly nutritious, easily digested and contains maternal 
cells and substances that act as a natural vaccine for the infant against a host of immunological threats including bacterial diarrhoea $^{(7,8)}$. Delivery of the placenta reduces physiological levels of oestrogen and progesterone, signalling the start of lactogenesis II and secretion of a more carbohydrate-rich and more fluid breast milk ${ }^{(6)}$, usually within 30-40 h postpartum. This increased fluid secretion dilutes the coloured immunological layer and makes it invisible to the naked eye. Because some women inaccurately perceive colostrum and later breast milk as two different substances, recognizing only the latter as true breast milk, they may delay breast-feeding until their breasts secrete the more fluid milk.

Colostrum avoidance includes: delayed initiation of breast-feeding; pumping and discarding colostrum; and/or wet nursing ${ }^{(9)}$. Some mothers in developing countries avoid colostrum feeding on the basis of traditional or cultural beliefs that range from viewing it as having no nutritional value, seeing it as harmful to the infant's health (making the baby sick or even causing death), or seeing it as 'bad luck' for the family ${ }^{(9-12)}$. Some women may specify no reason for avoiding colostrum other than tradition ${ }^{(13,14)}$.

Colostrum avoidance has been reported across the globe, in varied places such as India ${ }^{(15,16)}$, Nepal ${ }^{(17)}$, Bangladesh ${ }^{(13)}$, Indonesia $^{(18)}$, West Java ${ }^{(19)}$, the Philippines ${ }^{(20)}$, Vietnam ${ }^{(12)}$, Thailand $^{(21)}$, Bolivia ${ }^{(22)}$, Guatemala ${ }^{(14)}$, Guinea-Bissau ${ }^{(23)}$, Nigeria $^{(24)}$ and Tanzania ${ }^{(25)}$. Using the term 'delayed breast-feeding', Morse et $a l^{(9)}$ found that fifty of 120 cultures described in the Human Research Area Files (HRAF) 'withheld' colostrum. The extent to which colostrum is actually rejected has been questioned because of the methodological definitions of colostrum itself and terms such as 'colostrum rejection', 'withholding' and 'discarding ${ }^{, 26,27)}$. Nevertheless, the attention paid to the topic is evidence of the fact that any practice that withholds beneficial, readily available nutrition from infants is of concern.

The question of colostrum avoidance or discarding gains further importance when one considers that prelacteal foods are introduced when breast-feeding is delayed. Prelacteal substances may be given for non-nutritional reasons, such as to 'clear the throat' or bowels, or they may be traditional practices with no explicit intent ${ }^{(11,28)}$. Common prelacteals include unboiled cow/goat milk or sweetened water ${ }^{(9,19,23,25,29,30)}$; substances such as soda, cookies and bananas have also been reported ${ }^{(14,31)}$. All prelacteal substances have fewer nutrients and immunological components than colostrum and may introduce contaminants in the substances themselves or through finger feeding ${ }^{(11,32)}$.

Ethiopia has one of the highest infant mortality rates in the world and inappropriate neonatal feeding is a primary factor $^{(33)}$. Although breast-feeding is almost universal across Ethiopian ethnic groups and geographical areas, it does not always meet WHO/UNICEF recommendations. Data from the 2005 Ethiopian Demographic and Health
Survey ${ }^{(34)}$ show that this is true for groups living in the Amhara region that includes the village that was the subject of the present study (Fig. 1, area 3). Among all births in Ethiopia's eleven major regions between 2001 and 2005, Amhara mothers had the second lowest percentage of breast-feeding in the first hour after birth $(62.6 \%)$ and breast-feeding within $1 \mathrm{~d}$ of birth $(77 \cdot 4 \%$; Table 1). Less than half of Amhara infants (44.8\%) received colostrum, placing it the fourth lowest among eleven regions. A recent study ${ }^{(35)}$ compared breast-feeding practices in the Gondar province (an urban Amhara town) with those in the northernmost province of Tigray (Fig. 1, area 11). New mothers in Tigray were almost twice as likely as mothers in Gondar to initiate breastfeeding within the first hour of birth (Tigray $59 \%$ and Gondar 34.2\%).

A recent UNICEF press release on Ethiopian breastfeeding notes, 'Often, infants are not fed with colostrum ... as it is considered unclean'(30). The Ethiopian LINKAGES breast-feeding educational programme reports that mothers 'often discard colostrum'(36). A report from the Amhara region ${ }^{(37)}$ quotes a traditional birth attendant who describes her former belief that breast milk should not be used until the placenta is passed, which could take as long as $3 \mathrm{~d}$ in difficult births. Before the programme, the birth attendant endorsed the belief that the 'first milk' was 'bad' for the baby. Amhara mothers interviewed by Almedom ${ }^{(38)}$ noted a positive correlation between valuing colostrum and early breast-feeding initiation and reported a delay in breast-feeding 'when colostrum was considered "heavy"'. He also reported prelacteal feeding for all ethnic groups represented (Gurage, Oromo, Amhara, Sidama, Wellamo, Dorze) with one exception (Tigray) ${ }^{(38)}$.

Describing practices contributing to malnutrition during their 50 years of involvement in the rural Amhara village of Kossoye, members of the Kossoye Development Project

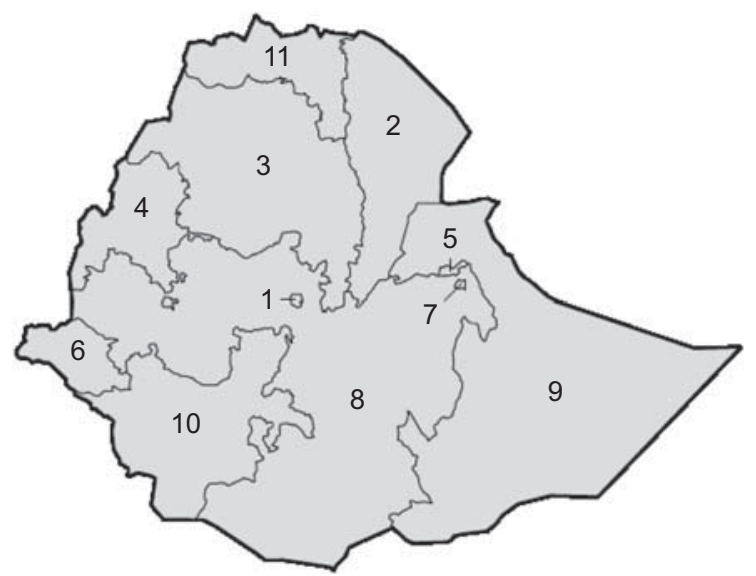

Fig. 1 Ethiopia administrative regions and chartered cities ${ }^{(58)}$ (1, Addis Ababa; 2, Afar; 3, Amhara; 4, Benishangul-Gumuz; 5, Dire Dawa; 6, Gambela; 7, Harari; 8, Oromiya; 9, Somali; 10, Southern Nations, Nationalities, and People's Region; 11, Tigray) 


\begin{tabular}{|c|c|c|c|c|}
\hline & $\begin{array}{l}\text { Breast-fed within } \\
1 \mathrm{~h} \text { of birth }\end{array}$ & $\begin{array}{l}\text { Breast-fed within } \\
1 \mathrm{~d} \text { of birth }\end{array}$ & $\begin{array}{l}\text { Received } \\
\text { prelacteal feed }\end{array}$ & $\begin{array}{l}\text { Received } \\
\text { colostrum }\end{array}$ \\
\hline Region & $\%$ & $\%$ & $\%$ & $\%$ \\
\hline Addis Ababa* & $66 \cdot 2$ & $86 \cdot 7$ & $49 \cdot 4$ & $42 \cdot 1$ \\
\hline Affar & $86 \cdot 4$ & $91 \cdot 1$ & $36 \cdot 8$ & $68 \cdot 3$ \\
\hline Amhara & $62 \cdot 6$ & $77 \cdot 4$ & $44 \cdot 6$ & $44 \cdot 8$ \\
\hline Benishangul-Gumuz & $72 \cdot 1$ & $80 \cdot 3$ & $19 \cdot 0$ & $45 \cdot 4$ \\
\hline Dire Dawa* ${ }^{*}$ & $91 \cdot 4$ & $94 \cdot 4$ & $34 \cdot 2$ & $66 \cdot 5$ \\
\hline Gambela & $72 \cdot 7$ & $80 \cdot 8$ & $28 \cdot 3$ & $43 \cdot 0$ \\
\hline Harari & $73 \cdot 7$ & $88 \cdot 2$ & $48 \cdot 6$ & $75 \cdot 9$ \\
\hline Oromiya & $72 \cdot 1$ & $88 \cdot 5$ & $26 \cdot 0$ & $45 \cdot 8$ \\
\hline Southern Nations, Nationalities and People's Region & $71 \cdot 4$ & $92 \cdot 7$ & $15 \cdot 4$ & $39 \cdot 3$ \\
\hline Somali & $91 \cdot 4$ & $94 \cdot 0$ & $19 \cdot 2$ & $53 \cdot 7$ \\
\hline Tigray & $52 \cdot 9$ & $73 \cdot 7$ & $30 \cdot 6$ & $55 \cdot 8$ \\
\hline
\end{tabular}

Adapted from Central Statistical Agency ${ }^{(34)}$, p. 144.

*Addis Ababa and Dire Dawa are cities, whereas the remaining nine areas are regional authorities.

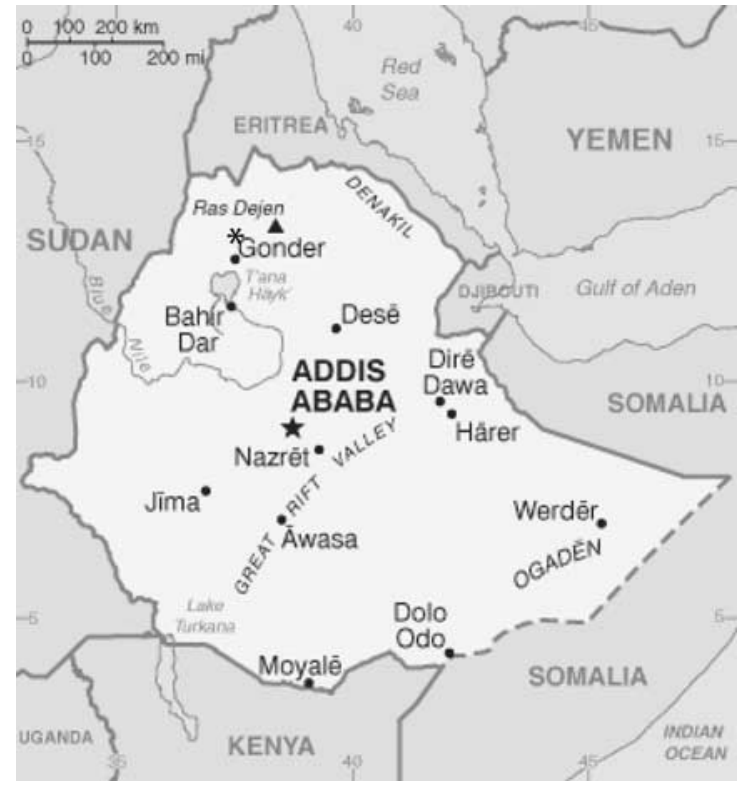

Fig. 2 The location of Kossoye $(*)^{(59)}$

(http://www.kossoyeproject.org/) note 'Colostrum ... is often discarded by mothers, ${ }^{\text {(39) }}$. As part of their recent initiative to reduce malnutrition in this village through sustainable organic gardening (2007-present), the Kossoye Development Project approved this preliminary investigation of infant feeding practices with the goal of identifying potential cultural and behavioural factors that might be influenced to increase colostrum feeding.

\section{Methods}

Kossoye is a rural village with associated hamlets located in the centre of the Northern Gondar province of the Amhara region (Fig. 2). The primary ethnic groups are self-described as Kemant or Amhara, two groups that have coexisted for centuries. They were historically differentiated by language and religion but long-term proximity has largely erased these differences ${ }^{(39)}$.

We hoped to somewhat mitigate the effect of the small sample size of individual maternal interviews by elucidating cultural norms from experienced native informants employed in the field of child and maternal health, all of whom live and work in the village. Although our findings may not be generalizable to other populations, our method may be useful to others wishing to conduct needs assessments in similar situations. The Kossoye Development Project hires villagers as community health workers (CHW) to conduct trainings and oversee the gardening project and its health- and nutrition-associated activities. Informal discussions were conducted with six of the female CHW and two awaledges (traditional birth attendants) currently practising in Kossoye. These participants were told of the study's goals and encouraged to ask any questions that arose during these discussions. In this manner, women working directly with new mothers and their families provided basic background information on childbirth, infant care and feeding, as well as cultural practices related to childhood growth and health.

For participants in the formal study, a semi-structured tape-recorded interview was developed using information from the literature and approved by the Wright State University Institutional Review Board. Informed consent documents were translated from English to Amharic and translated back to ensure accuracy. The structured interview included questions about parental age and education; mother's ethnicity; number of live births and age of children; breast-feeding behaviours in the first $3 \mathrm{~d}$ after birth through to weaning; availability and use of formula; and specific questions about colostrum $v$. other stages of breast milk. The semi-structured interview contained direct questions designed to evoke short answers and allow for immediate follow-up. Interviews were recorded and answers were translated into English by a clinically trained nurse midwife who is fluent in both Amharic and English (J.A.). 
Table 2 Age distribution of respondents' children*

\begin{tabular}{lccccccrrr}
\hline Age (years) & $<1$ & $1-1.9$ & $2-2.9$ & $3-3.9$ & $4-4.9$ & $5-9.9$ & $10-14.9$ & $15-19 \cdot 9$ & $\geq 20$ \\
\hline Youngest child $(n$ 20) & 4 & 5 & 3 & 3 & 1 & 2 & 0 & 1 \\
All children $(n 67)$ & 4 & 5 & 3 & 6 & 5 & 18 & 12 & 6 & 8 \\
\hline
\end{tabular}

${ }^{*}$ Age was not reported for eleven children.

CHW identified potential participants and we employed skilled native Ethiopian translators fluent in Amharic and English for interviews. Any woman reporting a live birth during her lifetime was eligible, and we specifically asked the CHW to introduce us to women with young children, women with older children and those with adult children to identify potential age-related differences in reported breast-feeding behaviour. Sixteen of the respondents reported that their youngest child was under the age of 5 years (Table 2 ).

Participants were read the informed consent in its entirety in the Amharic language and they agreed to participate before the initiation of recording. For individuals who were unable to write, an ink-stained fingerprint was used to indicate their agreement on the informed consent document in place of a signature. In exchange for their time, participants were given a stipend of five Ethiopian Birr ( $\sim 50$ cents US), roughly the price of a lunchtime meal in the local economy.

Seven of the twenty interviews were carried out under the auspices of Wright State University protocol, with N.L.R. as the interviewer. Using the same questionnaire and protocol, J.A. carried out an additional thirteen interviews under the auspices of the Kossoye Development Project. All interviews were conducted between May and July 2009 and lasted between 30 and $60 \mathrm{~min}$. No woman who was invited to participate refused. The two interviewers were both either present during the interviews or reviewed the content of the first seven interviews to build a consensus on points needing specific clarification and probing, especially given shades of meaning lost in translation.

\section{Results}

\section{Terms and identification of colostrum and breast milk}

Colostrum (inger) and breast milk (yetut wotet) are differentiated in the Amharic language; respondents identified colostrum as inger and later breast milk as yetut wotet. They noted that inger was produced earlier, was yellow or orange and was thicker than yetut wotet. Production of yetut wotet was indicated by breast heaviness in the $2-3 \mathrm{~d}$ following birth. The term makamesha was used for the traditional prelacteal feed of butter, cow's milk, ersho (yeast used to make the traditional Ethiopian bread injera) or some combination (e.g. butter and ersho).

\section{Discussions with community bealth workers}

The CHW host monthly discussions of health-related topics following a curriculum designed by The Kossoye Development Project and pay home visits to reinforce these messages. They are trained in and teach new mothers WHO-approved feeding practices, including exclusive breast-feeding from birth (including colostrum). To discourage the traditional practice of colostrum avoidance, $\mathrm{CHW}$ use the behaviour of mother cows to illustrate the natural nature of immediate breast-feeding: 'The cow gives her baby milk immediately after birth - why can't you?'

CHW told us that a new mother might travel to her mother's house to deliver and recuperate and her mother might come to her home for subsequent pregnancies to assist with the older children. New mothers learn about child care from their mothers and mothers-in-law, and from older female family members and respected neighbours. This reliance on family and community has perpetuated the practice of colostrum avoidance.

CHW state that breast-feeding is viewed positively in Kossoye and is seen as forming a strong bond between mother and baby. They said that if an older child is badly misbehaving or out of control, a mother might show her breast and tell the child 'I hurt from my breast' to remind them of that close relationship to pressurize them into changing their behaviour. The bond is further supported by routine bed sharing and the fact that the father may leave the marital bed for 6 weeks to 1 year to avoid sleep interruption.

\section{Discussions with traditional birth attendants}

The two awaledges interviewed for the study were actively engaged in delivering children in the Kossoye area. Both categorized colostrum as being different from breast milk. They noted that mothers usually initiate breast-feeding on day 2 to stimulate breast milk, which would then become available on day 3. The awaledges claim that new mothers traditionally learnt about breast-feeding from family members or trusted neighbours, and hence they seldom provided feeding instructions after delivery. Therefore, they neither recommend nor discourage discarding or avoiding colostrum. They did, however, endorse and conduct makamesha (prelacteal feeding of newborns). One attendant reported that butter might be fed to girls using a gold-coloured spoon and to boys using a silvercoloured spoon. There was no general consensus about symbolism of the different coloured metals and it was primarily discussed as a pleasant tradition. 


\section{Interviews with village women}

A slight majority of the twenty women interviewed selfidentified as Kemant than Amhara (Table 3). The age of participants ranged from 20 to 60 years. Male partners were older than their female partners with one exception in which the partners were of the same age. Mothers were more likely than their male partners to be illiterate. The number of reported live births ranged from 1 through 8 , with a mean of just under 4 .

All but one of the respondents gave birth to their children at home with the aid of an awaledge or family member and breast-fed their children. One woman delivered at a clinic and subsequently fed her child formula. However, she reported no problems with pregnancy or delivery and it appears that her employment in a city allowed her to attend a medical clinic. Four of the twenty women interviewed (20\%) were aware of the contraceptive effect of breast-feeding; the remainder saw breast-feeding as being beneficial only to the infant.

A total of $79 \%$ of the women who breast-fed (fifteen of nineteen) reported discarding colostrum before breastfeeding. However, the same percentage also claimed to initiate breast-feeding within $24 \mathrm{~h}$ of birth (Fig. 3). The women's descriptions make it clear that only a portion of the colostrum was discarded before breast-feeding. This

Table 3 Summary of Kossoye respondents

\begin{tabular}{lrrr}
\hline & Mean & SD & Range \\
\hline Age (years) & & & \\
Woman & & & \\
$\quad$ Partner & $32 \cdot 26$ & $11 \cdot 49$ & $20-60$ \\
Number of live births & $36 \cdot 89$ & $10 \cdot 06$ & $24-66$ \\
& $3 \cdot 85$ & $2 \cdot 03$ & $1-8$ \\
& Illiterate & Literate & Grades \\
\cline { 2 - 3 } & & & \\
Education & 13 & 7 & $4-10$ \\
$\quad$ Women & 8 & 12 & $2-10$ \\
Partner & $n$ & $\%$ & \\
& & & \\
Ethinicity & 12 & 60 & \\
$\quad$ Kemant & 8 & 40 & \\
Amhara & & & \\
\hline
\end{tabular}

*Age was not known for one woman and two partners.

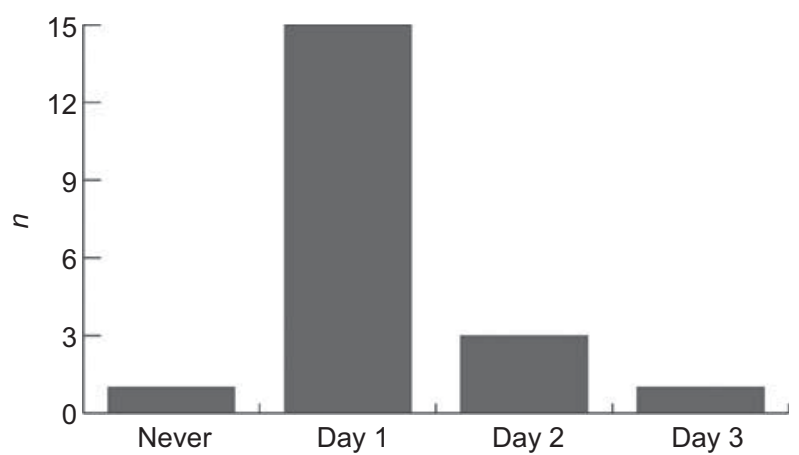

Fig. 3 Breast-feeding initiation in Kossoye practice was continued by some until they perceived an increase in fluid volume in their breasts and/or breast milk.

The consensus view was that colostrum was of potential harm to newborns. Nine women specifically referred to abdominal cramps or stomach ache as a consequence of feeding inger, two specified diarrhoea, three said that it contaminated an infant's stomach and two thought it would introduce the Ascaris parasite. One woman called it 'bad for the stomach' and another said it was 'our culture' to dispose of inger. Inger was described as 'dirty milk' by one mother and another suggested that a child who ingested inger might eventually die from its effects (some interviewees referred to more than one consequence).

Makemesha was described as a traditional practice associated with birth and was reported by twelve of the nineteen women who breast-fed. The most common prelacteal foods described were butter ( $n$ 4), butter and/or ersho ( $n 4)$ and cow's milk ( $n 4)$, which might be diluted with water. This food was offered in order to ensure that the first thing the baby tasted was smooth and to 'smooth the throat' (this was described as referring to the oesophagus rather than the larynx, with no reference to the baby's voice). The woman who endorsed an actual substitution of butter for colostrum until after day 3 was from a generation older than those who endorsed only a ritual prelacteal feed and had only adult children.

\section{Discussion}

\section{Colostrum avoidance}

Despite the linguistic differentiation of colostrum (inger) and breast milk (yetut wotet) and the support for avoiding colostrum, the majority of women initiated breast-feeding within $24 \mathrm{~h}$ of birth. The description of practices leads us to infer that discarding a portion of the colostrum is seen as being sufficient to cleanse the breast of the unwanted substance and/or its perceived negative consequences. It is unclear how much of the colostrum is discarded overall or how often mothers nurse in the first $3 \mathrm{~d}$ postpartum. These factors would need to be directly observed and assessed to accurately evaluate the functional significance of the practice on neonatal health. Additional study is planned to assist in developing appropriate interventions.

Kossoye's traditional belief that colostrum may cause abdominal discomfort and diarrhoea conflicts with the fact that it protects abdominal health by decreasing permeability of the gut to infectious substances ${ }^{(27)}$. Further, evidence indicates that growth hormones of the colostrum nurture the endothelium in the neonate's gastrointestinal tract in the first days and months of life ex utero ${ }^{(40)}$. This is in addition to the many additional immunological substances and protections that colostrum provides ${ }^{(7,8)}$.

The present study supports Holman and Grimes, (41) proposal that, despite the widespread reporting of colostrum taboos, total avoidance is 'rare'. Rizvi ${ }^{(27)}$ suggests that 
many early studies may have based their conclusion of colostrum avoidance on the practice of prelacteal feeding, which does not per se rule out early initiation of breastfeeding. However, as the proportion of immunological substances is greatest in the first $3 \mathrm{~d}$ following birth $^{(27,42)}$, any practice that reduces the frequency or volume of breast-feeding during this time could reduce an infant's long-term health and immunological defence.

\section{Prelacteal feeding}

Prelacteal feeding emerged as a maladaptive practice that was not previously identified as a target for intervention. It is equally important to neonatal health as colostrum feeding. Further, because it takes place before the beneficial actions of the colostrum on the gut, unpasteurized substances are introduced when the gut is at its most permeable and vulnerable to contaminants. The calculated rate of $60 \%$ prelacteal feeding in Kossoye is intermediate among reported prevalence rates, which range from approximately $15 \%{ }^{(12,17)}$ to $100 \%{ }^{(24,27)}$. Getahun et al. ${ }^{(35)}$ report that unsalted butter is given to newborns in Gondar (20 miles south of Kossoye) 'in the belief that it will clean the stomach'. In that study, $62 \%$ of Gondarine newborns received butter in the first days of life, and rural mothers were significantly more likely than those in urban Gondar to give prelacteal butter $(P<0 \cdot 0007)$. Another Ethiopian study reported that prelacteal fluids were thought to 'clean the baby's throat' and were given over the first $3 \mathrm{~d}$ of birth ${ }^{(2)}$, longer than the Kossoye practice. The primary dangers of prelacteal feeding are: (i) no prelacteal feed provides immunological benefits equal to colostrum; (ii) prelacteal substances may contain harmful contaminants; (iii) some prelacteals are semi-solids that infants are unable to digest; (iv) prelacteal feeding may encourage delayed breastfeeding initiation; and (v) prelacteal feeding and handling of the baby immediately after birth disrupt the mother/baby dyad, causing stress in both the mother and the baby ${ }^{(43)}$.

The complementary relationship of prelacteal feeding and delayed breast-feeding initiation has been called a 'vicious cycle' because prelacteal feeding may delay the production of breast milk that the mother perceives to be 'mature', and the perceived lack of 'mature' breast milk may encourage the use of prelacteal foods ${ }^{(44)}$. Contrary to these reports of the vicious cycle, makamesha in Kossoye does not appear to prevent a majority of mothers from initiating breast-feeding within $24 \mathrm{~h}$ of birth. This is likely because mothers explain that nutrition is not makamesha's primary purpose. Getahun et $a l^{(35)}$ report a similar coincident practice of prelacteal feeding and colostrum feeding in nearby Gondar, noting that $68.5 \%$ of newborns experienced both. The primary danger of contamination argues for an intervention that discourages prelacteal feeding ${ }^{(43,45)}$.

Despite the encouraging finding that most infants in Kossoye receive some colostrum, in the context of Ethiopian growth deficits it is imperative that breast-feeding behaviour be maximized to meet WHO/UNICEF standards.
The immune potential of the colostrum is at its peak in the first $24 \mathrm{~h}$ following birth $^{(27,42)}$, containing up to twelve times the secretory IgA and more than twice the lactoferrin of mature milk ${ }^{(46)}$. Recent studies of neonatal mortality in rural Ghana found that delayed breast-feeding initiation of only $1 \mathrm{~d}$ conferred a $2 \cdot 4$-fold increase in the risk of death $^{(47)}$. The authors estimate that the neonatal mortality rate could be reduced by $16 \%$ if breast-feeding was initiated on the day of birth and by $22 \%$ if started in the first hour ${ }^{(47)}$. Despite the infective danger posed by prelacteal feeding, it was not statistically associated with increased mortality in that study. However, the potential for introducing contaminated substances to neonates still argues against even ritual prelacteal feeding.

Possible intervention in Kossoye is simplified because no religious basis was reported for either colostrum avoidance or prelacteal feeding. Nevertheless, it is possible that cultural expectations of postpartum fatigue and pain may prevent the universal acceptance of immediate breast-feeding ${ }^{(9,27)}$. Value of the makamesha tradition may also be a considerable barrier. Another concern is maintaining adherence to an intervention's recommendations. Given that even trained medical professionals may not encourage immediate breast-feeding ${ }^{(21)}$, maintaining fidelity to recommendations among lay health advisors and the public may be an even greater challenge. The results of a successful educational intervention in India note that periodic reinforcement of nutritional information may be necessary to sustain and/or improve behavioural change ${ }^{(16)}$.

\section{Conclusion and recommendations}

Recent international initiatives have drawn the attention of Ethiopian mothers to exclusive breast-feeding in the first 6 months of life ${ }^{(30,32)}$, and the Ethiopian Federal Ministry of Health trains its health workers to educate new mothers about internationally recommended feeding practices. The World Bank labels this type of intervention as a 'short route' to better nutrition because change can be achieved more quickly through smaller-scale interventions than through infrastructural and policy interventions $^{(48)}$. We commend these educational efforts and suggest that local awaledges be specifically recruited to augment current educational initiatives, implement postpartum practices that support the mother/infant dyad, encourage immediate breast-feeding of colostrum after birth and discourage all prelacteal feeding. Traditional birth attendants have been shown to significantly increase proactive health behaviours in expectant and new mothers ${ }^{(49,50)}$. As respected members of the community, they are important mediators of the relationship between the formal health community and the target population $^{(51)}$. They attend the great majority of births $(94 \%$ of Ethiopian deliveries between 2001 and 2005 were at 
home ${ }^{(34)}$ ) and are therefore well placed to assist new mothers in their first breast-feeding. It may also be helpful to add colostrum feeding to the child's immunization card to further underscore its immunological benefits. This practice was adopted by Curamericas in Guatemala ${ }^{(14)}$ because the mothers already accepted the importance of immunizations and the association of this concept with colostrum was expected to increase colostrum feeding.

Culturally tailored educational programmes conducted in women's groups using community health advocates and traditional health practitioners can successfully change even long-standing traditions. For example, an Indonesian programme increased neonatal colostrum feeding by $132 \%^{(18)}$ and a case-control study in Nepal showed a significant positive change in colostrum feeding, antenatal care and placental cord care ${ }^{(52)}$. A colostrum feeding intervention could be integrated into the Kossoye Development Project's ongoing efforts to reduce malnutrition with an expectation of success given the project's 50-year relationship with the community and successful interventions for trachoma ${ }^{(39)}$ and gardening. The potential of success is also supported by a study by the LINKAGES breast-feeding programme (2003-2006). Using the Essential Nutrition Actions approach that included optimal breast-feeding education, they reported a $37 \%$ increase $(P>0 \cdot 001)$ in first-hour breast-feeding among participating Amhara mothers ${ }^{(36)}$.

It is important for village women of all ages to be educated about colostrum and prelacteal feeding, given the strong influence of older generations ${ }^{(13,53-55)}$. This will further empower women to realize their central importance to community health, eloquently stated by Shirima et $a l .{ }^{(56)}$ :

...there is a need to inform the mothers as well as society as a whole about the advantages conferred to the mother and the community by breast-feeding. Such information may help the community to acknowledge the contribution of the mother to the family and nation through breast-feeding and inspire them to help create the circumstances necessary for her to be able to breast-feed successfully.

Using information from this ethnographic enquiry, we propose that a cooperative relationship between the traditional and formal health systems is a culturally competent solution that aligns with the Ethiopian government's staunch support for traditional medicine (reviewed in Kassaye et al. ${ }^{(57)}$ ) and its need for improved health outcomes.

\section{Acknowledgements}

The present study was supported by Wright State University's Substance Abuse Resources and Disability Issues Program and the Kossoye Development Project. The authors have no financial conflict of interest to report. N.L.R., A.J.C. and D.C. are members of the Kossoye Development Project Board and J.A. was a paid consultant for the project. N.L.R., D.M., S.N., A.J.C. and D.C. are credited with conceptualizing and designing the study; N.L.R., J.A., S.N., A.J.C. and D.C. with logistics and fieldwork; N.L.R., J.A., D.M., L.J.S., A.J.C. and D.C. with data analysis and interpretation; and D.M., A.J.C. and D.C. with acquisition of funds. All authors are credited with manuscript preparation.

\section{References}

1. Silva P (2005) Environmental Factors and Children's Malnutrition in Ethiopia: Policy Research Working Paper Series no. 3489. Washington, DC: The World Bank.

2. Alemayehu T, Haidar J \& Habte D (2009) Determinants of exclusive breastfeeding practices in Ethiopia. Ethiop $J$ Health Dev 23, 12-18.

3. Mannel R, Martens P \& Walker M (editors) (2007) Core Curriculum for Lactation Consultant Practice, 2nd ed. Sudbury, MA: Jones and Bartlett Publishers.

4. World Health Organization/UNICEF (2009) Baby-Friendly Hospital Initiative: Revised Updated and Expanded for Integrated Care. Geneva: WHO/UNICEF \& Wellstart International.

5. Vallenas C \& Savage F (1998) Evidence for the Ten Steps to Successful Breastfeeding. Geneva: WHO Division of Child Health and Development.

6. Kent J (2007) How breastfeeding works. J Midwifery Womens Health 52, 564-570.

7. Hanson L (2004) The Immunobiology of Human Milk: How Breastfeeding Protects Babies. Amarillo, TX: Pharmasoft Publishing.

8. Labbok M, Clark D \& Goldman A (2004) Breastfeeding: maintaining an irreplaceable immunological resource. Nature Rev 4, 565-572.

9. Morse J, Jehle C \& Gamble D (1990) Initiating breastfeeding: a world survey of the timing of postpartum breastfeeding. Int J Nurs Stud 27, 303-313.

10. Bhale P \& Jain S (1999) Is colostrum really discarded by Indian mothers? Indian Pediatr 36, 1069-1070.

11. Goyle A, Jain P, Vyas S et al (2004) Colostrum and prelacteal feeding practices followed by families of pavement and roadside squatter settlements. Indian J Prev Soc Med 25, 58-62.

12. Duong D, Binns C \& Lee A (2004) Breast-feeding initiation and exclusive breast-feeding in rural Vietnam. Public Health Nutr 7, 795-799.

13. Littler C (1997) Beliefs about colostrum among women from Bangladesh and their reasons for not giving it to the newborn. Midwives 110, 3-7.

14. Derewicz M (2007) Mother's Golden Milk. Chapel Hill, NC: University of North Carolina Global.

15. Agrawal D, Agrawal K \& Khare B (1985) Study on current status of infant and childhood feeding practices. Indian Pediatr 22, 716.

16. Galhotra A, Abrol A, Agarwal N et al. (2008) Impact of community based awareness campaign on breast-feeding among lactating women in Chandigarh. Internet J Health 7.

17. Osrin D, Tumbahangphe K, Shrestha D et al. (2002) Cross sectional, community based study of care of newborn infants in Nepal. BMJ 325, 1063-1066.

18. Wiryo $\mathrm{H} \&$ Hakimi $M$ (2005) Implementation of health education, based on ethnographic study, to increase the colostrum and decrease early solid food feeding. Health Educ Behav 32, 102-112. 
19. Utomo B, Fitria L, Sulacha E et al. (2000) Feeding patterns, nutrient intake and nutritional status among children 0-23 months of age in Indramayu, West Java, 1997. Mal J Nutr 6, 147-170.

20. Adair L \& Popkin B (1996) Low birth weight reduces the likelihood of breast-feeding among Filipino infants. $J$ Nutr 126, 103-112.

21. Kaewsarn P, Moyle W \& Creedy D (2003) Traditional postpartum practices among Thai women. $J$ Adv Nurs $\mathbf{4 1}$, 358-366.

22. Ludvigsson J (2003) Breastfeeding intentions, patterns, and determinants in infants visiting hospitals in La Paz, Bolivia. BMC Pediatr 3, 5-16.

23. Gunnlaugsson G \& Einarsdottir J (1993) Colostrum and ideas about bad milk: a case study from Guinea-Bissau. Soc Sci Med 36, 283-288.

24. Okolo S, Adewunmi Y \& Okonji M (1999) Current breastfeeding knowledge, attitude, and practices of mothers in five rural communities in the savannah region of Nigeria. I Trop Pediatr 45, 323-326.

25. Hussein A (2005) Breastfeeding and complementary feeding practices in Tanzania. East Afr J Public Health 2, 27-31.

26. Bansal RK \& Sitaraman S (1988) Colostrum deprivation: a misconception. Indian Pediatr 25, 396-397.

27. Rizvi N (1993) Issues surrounding the promotion of colostrum feeding in rural Bangladesh. Ecol Food Nutr 30, 27-38.

28. Engebretsen I, Wamani H, Karamagi C et al. (2007) Low adherence to exclusive breastfeeding in Eastern Uganda: a community-based cross-sectional study comparing dietary recall since birth with 24-hour recall. BMC Pediatr 7, 10-22.

29. Wamani H, Astrom A, Peterson S et al. (2005) Infant and young child feeding in Western Uganda: knowledge, practices and socio-economic correlates. J Trop Pediatr 51, 356-361.

30. UNICEF (2009) News Note: World Breastfeeding Week Celebrated in Ethiopia. New York: UNICEF.

31. Schmidt M, Muslimatum S, West C et al. (2002) Nutritional status and linear growth of Indonesian infants in West Java are determined more by prenatal environment than by postnatal factors. J Nutr 132, 2202-2207.

32. UNICEF (2004) News Note: FMOH and UNICEF Join Forces to Promote Safe Breastfeeding. New York: UNICEF.

33. Ministry of Health, Family Health Department (2004) National Strategy for Infant and Young Child Feeding. Addis Ababa: FMOH.

34. Central Statistical Agency (2006) Ethiopia Demographic and Health Survey 2005. Addis Ababa and Calverton, MD: CSA and ORC Macro.

35. Getahun Z, Scherbaum V, Taffese Y et al. (2004) Breastfeeding in Tigray and Gonder, Ethiopia, with special reference to exclusive/almost exclusive breastfeeding beyond six months. Breastfeed Rev 12, 8-16.

36. Academy for Educational Development, LINKAGES (2006) Breastfeeding, LAM, Related Complementary Feeding, and Maternal Nutrition Program in Ethiopia. LINKAGES/Ethiopia Final Report, 2003-2006. Washington, DC: AED LINKAGES.

37. John Snow Inc. (2009) Ethiopia: Promoting Breastfeeding to Ensure Healthier Babies. Boston, MA: JSI.

38. Almedom A (1991) Infant feeding in ubran low-income households in Ethiopia: I. The weaning process. Ecol Food Nutr 25, 97-109.

39. Carlson A \& Carlson D (2008) Health, Wealth and Family in Rural Ethiopia: Kossoye, North Gondar Region, 1963-2007. Addis Ababa: Addis Ababa University Press.
40. Xu RJ (1996) Development of the newborn GI tract and its relation to colostrum/milk intake: a review. Reprod Fertil Dev 8, 35-48.

41. Holman DJ \& Grimes MA (2001) Colostrum feeding behaviour and initiation of breast-feeding in rural Bangladesh. J Biosoc Sci 33, 139-154.

42. Zarban A, Taheri F, Chahkandi T et al. (2009) Antioxidant and radical scavenging activity of human colostrum, transitional, and mature milk. J Clin Biochem 45, 150-154.

43. Groer M \& Davis M (2002) Postpartum stress: current concepts and the possible protective role of breastfeeding. J Obstet Gynecol Neonatal Nurs 31, 411-417.

44. Athavale A, Athavale S, Deshpande S et al. (2004) Initiation of brest-feeding by urban women. Health Popul Perspect Issues 27, 117-125.

45. Marín G, Llana M, López E et al. (2009) Randomized controlled trial of early skin-to-skin contact: effects on the mother and the newborn. Acta Paediatr 99, 1630-1634.

46. Hanson L (2004) Mother's defense of the offspring via the milk. In Immunobiology of Human Milk: How Breastfeeding Protects Babies, pp. 77-122. Amarillo, TX: Pharmasoft Publishing.

47. Edmond K, Zandoh C, Quigley M et al. (2006) Delayed breastfeeding initiation increases risk of neonatal mortality. Pediatrics 117, e380-e386.

48. The World Bank (2006) Repositioning Nutrition as Central to Development: A Strategy for Large-Scale Action. Washington, DC: The World Bank.

49. Okubagzhi $G$ (1988) Fulfilling the potential of traditional birth attendants. World Health Forum 9, 426-431.

50. Isenalumbe A (1990) Integration of traditional birth attendants into primary health care. World Health Forum 11, 192-198.

51. Pose B, Abdullah M, Buchanan S et al. (2007) Rethinking the Role of Traditional Birth Attendants (TBAs) in Rural Communities - Outcomes from a Study in Ethiopia: Abstract 152751: APHA. Washington, DC: AJPH.

52. Wade A, Osrin D, Shrestha B et al. (2006) Behavior change in perinatal care practices among rural women exposed to a women's group intervention in Nepal. BMC Pregnancy Childbirth 6, 20-30.

53. Meikandan D (1987) Wastage of colostrum. Indian Pediatr 24, 952-953.

54. Yadav R \& Singh P (2004) Knowledge, attitude and practices of mothers about breast-feeding in Bihar. Indian J Community Med 29, 7-9.

55. Masvie H (2006) The role of Tamang mothers-in-law in promoting breast feeding in Makwanpur District, Nepal. Midwifery 22, 23-31.

56. Shirima R, Greiner T, Kylberg E et al. (2001) Exclusive breast-feeding is rarely practiced in rural and urban Morogoro, Tanzania. Public Health Nutr 4, 147-154.

57. Kassaye K, Amberbir A, Getachew B et al. (2006) A historical overivew of traditional medicine practices and policy in Ethiopia. Ethiop J Health Dev 20, 127-134.

58. Wikipedia (2005) File:Ethiopia regions numbered.png. Created by User:Goldberg, Wikipedia administrator. Licensed under the Creative Commons Attribution 2.0 Generic License. http:// www.wikipedia.org/wiki/File:Ethiopia_regions_numbered.png (accessed March 2011).

59. US Department of State, Bureau of African Affairs (2010) Background Note: Ethiopia. http://www.state.gov/r/pa/ei/ bgn/2859.htm (accessed March 2011). 